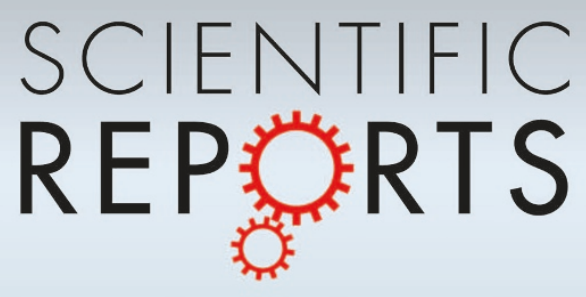

OPEN

SUBJECT AREAS:

COMPLEX NETWORKS

NONLINEAR PHENOMENA

Received

12 July 2013

Accepted

18 February 2014

Published

7 March 2014

Correspondence and requests for materials should be addressed to S.S. (sudeshna@ iisermohali.ac.in)

\section{Taming Explosive Growth through Dynamic Random Links}

\author{
Anshul Choudhary, Vivek Kohar \& Sudeshna Sinha
}

Indian Institute of Science Education and Research (IISER) Mohali, Knowledge City, SAS Nagar, Sector 81, Manauli PO 140306 , Punjab, India.

We study the dynamics of a collection of nonlinearly coupled limit cycle oscillators relevant to a wide class of systems, ranging from neuronal populations to electrical circuits, over network topologies varying from a regular ring to a random network. We find that for sufficiently strong coupling strengths the trajectories of the system escape to infinity in the regular ring network. However when a fraction of the regular connections are dynamically randomized, the unbounded growth is suppressed and the system remains bounded. Further, we find a scaling relation between the critical fraction of random links necessary for successful prevention of explosive behavior and the network rewiring time-scale. These results suggest a mechanism by which blow-ups may be controlled in extended oscillator systems.

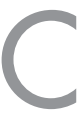

ollections of coupled dynamical systems were introduced as simple models capturing the essential features of large interactive systems ${ }^{1}$. Research focused on such distributed systems and complex networks has provided frameworks for understanding spatiotemporal patterns in problems ranging from Josephson junction arrays and multimode lasers, to microfluidic arrays and evolutionary biology ${ }^{2-5}$.

In this work we study the dynamics of nonlinearly coupled oscillators relevant to systems ranging from neuronal populations to electrical circuits, over network topologies varying from a regular ring to a random network. We find that for sufficiently strong coupling strengths the trajectories of this system escape to infinity in the regular ring topology, i.e. the amplitude of the oscillations grows explosively without bound. We term this phenomenon a blow-up. The surprising central result of this work is the following: the unbounded growth is suppressed when some of the regular connections are dynamically randomized. Namely, dynamic random links successfully reign in the blow-ups in this complex network, and normal limit cycle behavior ensues for sufficiently fast network rewiring.

This counter-intuitive result is significant in the general context of taming infinities, as infinities arising in theoretical constructs of physical phenomena deeply concern scientists across disciplines ${ }^{6}$. When physical quantities tend to infinite values in a model, it is often considered an indication of the failure of the theory, or symptoms of the limitations of the modeling. Removing infinities, for instance through theoretical tools such as renormalization, or through improvement of the underlying model of the physical process, has had much impact on physics in general. Further from the point of view of potential applications it is indeed crucial that no quantity grows without bound. So the drive to find mechanisms that can quell infinities in measurable quantities stems from both methodological and pragmatic motivations ${ }^{6}$. Specifically, our observations in this work may yield methods to control and prevent blow-ups in extended oscillator systems which are widely employed in a multitude of engineered devices. Lastly, a certain degree of randomness in spatial links may be closer to physical reality in many systems of biological, technological and physical significance ${ }^{7}$. So our finding that random links prevent unphysical blow-ups suggests an underlying mechanism by which complex systems can avoid explosive growths.

\section{Results}

We start with a generic coupled system comprised of nonlinear local dynamics and a term modeling the coupling interaction. The isolated (uncoupled) dynamics at each node of the lattice/network is given by $\dot{X}=\mathrm{F}(\mathrm{X})$, where $\mathrm{X}$ is a $m$-dimensional vector of dynamical variables and $F(X)$ is the velocity field. The general form of such coupled dynamical elements is given by following equation:

$$
\dot{\mathrm{X}}_{i}=\mathrm{F}\left(\mathrm{X}_{i}\right)+\epsilon \sum_{j=1}^{N} J_{i j} \mathrm{H}\left(\mathrm{X}_{i,} \mathrm{X}_{j}\right), \quad i=1, \ldots, N,
$$



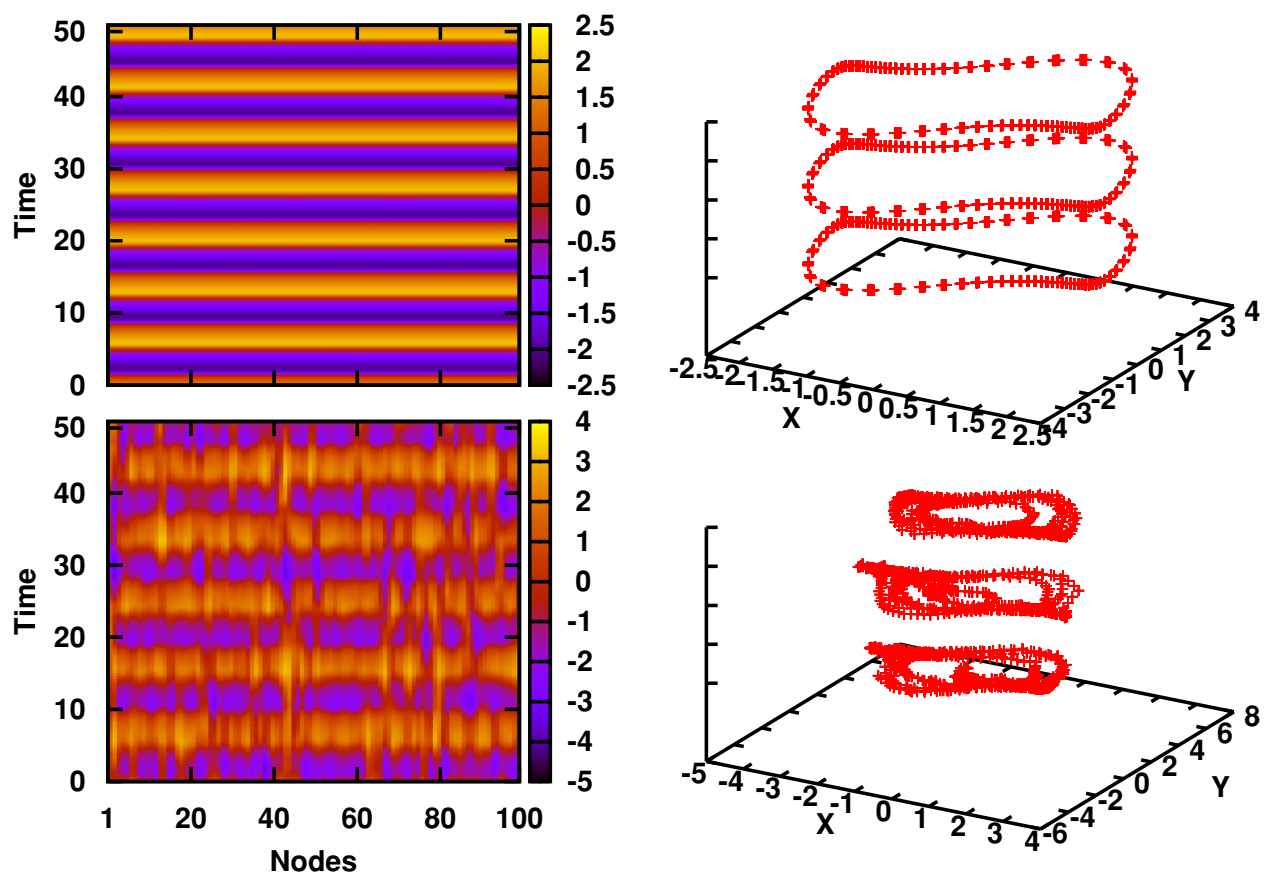

Figure $1 \mid$ Evolution of $x_{i}(t)(i=1, \ldots 100)$, after a transient time of 15000 (left), and the limit cycles of three representative oscillators stacked one above the other (right), for network rewiring period r equal to 0.01 (top row) and 0.1 (bottom row). Here coupling strength $\epsilon=0.6$ which is significantly greater than critical coupling strength $\left(\epsilon_{c}=0.5\right.$ for these parameter values) and the fraction of random links $p$ is 0.8 . Blow-up is prevented in both the cases, but faster rewiring leads to a synchronized state (top row) whereas slower rewiring leads to de-synchronization and distortion in the shape of the limit cycles (right panel, bottom row). Note that for this value of coupling strength, regular nearest neighbor coupling $(p=0)$ would lead to a blow-up.

where $J_{i j}$ are the elements of a connectivity matrix, and need not in general be symmetric ${ }^{8}$. The coupling strength is given by $\epsilon$ and $\mathbf{H}\left(\mathbf{X}_{i}\right.$, $\mathbf{X}_{j}$ ) is the coupling function determined by the nature of interactions between dynamical elements $i$ and $j$.

At the nodes, we first consider the prototypical Van der Pol oscillator ${ }^{9,10}$ with nonlinear damping governed by the second-order differential equation:

$$
\ddot{x}+\mu\left(x^{2}-1\right) \dot{x}+x=0
$$

where $x$ is the dynamical variable and $\mu$ is a parameter which determines the nature of the dynamics. The relaxation oscillations arising in this system, for $\mu>0$, are characterized by slow asymptotic behavior and sudden discontinuous jumps. Associating $\dot{x}=y$ gives:

$$
\begin{aligned}
& \dot{x}=f(x, y)=y \\
& \dot{y}=g(x, y)=-\mu\left(x^{2}-1\right) y-x
\end{aligned}
$$

The Van der Pol equation given above is relevant in modeling phenomena in physical and biological sciences. Extensions of such oscillators have been used to model the electrical activity of the heart and the action potentials of neurons ${ }^{11}$. The equation has also been utilised in seismology to model the two plates in a geological fault ${ }^{14}$.

Now we consider a ring of $N$ nonlinearly coupled Van-der-Pol oscillators $^{15-20}$, namely a specific form of Eqn. 1 with $m=2, \mathbf{X}_{i}=$ $\left\{x_{i}, y_{i}\right\}, \mathbf{F}=\{f, g\}$ and $\mathbf{H}\left(\mathbf{X}_{i}, \mathbf{X}_{j}\right)=\left\{f\left(x_{j}, y_{j}\right)-f\left\{\left(x_{i}, y_{i}\right), g\left(x_{j}, y_{j}\right)-g\left(x_{i}\right.\right.\right.$, $\left.\left.y_{i}\right)\right\}$ given as follows:

$$
\begin{aligned}
& \dot{x}_{i}=f\left(x_{i}, y_{i}\right)+\frac{\epsilon}{2}\left[f\left(x_{i-1}, y_{i-1}\right)+f\left(x_{i+1}, y_{i+1}\right)-2 f\left(x_{i}, y_{i}\right)\right] \\
& \dot{y}_{i}=g\left(x_{i}, y_{i}\right)+\frac{\epsilon}{2}\left[g\left(x_{i-1}, y_{i-1}\right)+g\left(x_{i+1}, y_{i+1}\right)-2 g\left(x_{i}, y_{i}\right)\right]
\end{aligned}
$$

Here index $i$ specifies the site/node in the ring, with the nodal on-site dynamics being a Van der Pol relaxation oscillator. Starting with this regular ring, we construct small world networks ${ }^{7}$ by rewiring with probability $p(0 \leq p \leq 1)$, the nearest neighbor links of a node to some non-local node chosen at random. Additionally the links are dynamic, which implies that the specific set of nearest neighbour links that get rewired to random nodes varies from time to time. So the underlying web of connections changes over time ${ }^{21-24}$, and such dynamic rewiring is expected to be widely prevalent in response to environmental influences or internal adaptations ${ }^{25-27}$. We implement this by rewiring the network at time intervals of $r$ (see methods). So a new connectivity matrix having the same proportion of random and nearest neighbor connections is formed after every time $r$. So $r$ gives the time-scale of network switches and as $r$ becomes larger the network changes become more infrequent, with the limit of $r \rightarrow \infty$ corresponding to the standard scenario of quenched links.

In order to get the dynamics of the complete system, we solved the $2 N$ coupled non-linear ordinary differential equations (ODE) given by Eqns. 3-4 with dynamic network topologies for various coupling strengths and system sizes. We studied the shapes and sizes of the limit cycles by varying the fraction of random links $p$, and network switching time period $r$.

We first describe our principal observations for oscillators coupled to two nearest neighbors on a periodic lattice (i.e. $p=0$ ) for various coupling strengths. When the coupling strength is between 0 and a critical coupling strength $\epsilon_{c}$, the system yields regular bounded behavior. However when the coupling strength exceeds this value, i.e. $\epsilon>$ $\epsilon_{c}$, there is a blow-up in the system. Namely, the amplitude of the oscillations grows explosively in an unbounded fashion ${ }^{20,28-32}$. For instance, in a typical blow-up the amplitude grows from $O$ (1) to $O\left(10^{4}\right)$ in time $\sim 10^{-3}$.

Now, very different behavior from that described above ensues when the nearest neighbor links are dynamically rewired. We show the results for representative values of $p$ and $r$ in Figure 1. Notice that the coupling strength shown in the figure is greater than the critical value $\epsilon_{c}$, i.e. for this coupling strength the limit cycles grow explosively for regular nearest neighbor coupling. However, it is clearly 
evident from the figure that the blow-up has been tamed when some fraction of the nearest neighbor links are replaced by random links (i.e $p>0$ ). Namely, for sufficiently random networks all oscillators remain confined in a bounded region of phase space.

To quantitatively characterize the bounded-unbounded transitions, we introduce a "boundedness order parameter" $Z_{\text {bound }}$ given simply as the fraction of initial conditions for which the dynamics remains bounded. Thus $Z_{\text {bound }}$ gives the probability of successful prevention of blow-ups. Fig. 2 displays this quantity over a range of $p$ and $\epsilon$. It is evident from Fig. 2 that we always get a bounded state if the fraction of random links $p$ is greater than a critical value.

The fraction of random links necessary to obtain bounded dynamics with probability close to 1 depends on the network switching time period $r$ and coupling strength $\epsilon$ as indicated in Fig. 2. In general, for fast switching topologies, namely small $r$, the critical number of random links needed to suppress a blow-up is small. However, for very slow network changes one may not be able to quell explosive growth even with large fraction of random links. So it is clear that rapidly changing links are the key to effectively control the dynamics.

Further, we studied the case of undirected networks (where $J$ in Eqn. 1 is a symmetric matrix). Qualitatively similar results are obtained though with reduced range for blow-up control (see lower panel of Fig. 2).

To broadly rationalize this behavior we consider the dynamics of an individual oscillator in the system as a sum of the nodal dynamics and the coupling contributions of the form $c_{1}(t)$ and $c_{2}(t)$, whose strength is given by coupling constant $\epsilon$.

$$
\begin{aligned}
& \dot{x}=y+\epsilon c_{1}(t) \\
& \dot{y}=-\mu\left(x^{2}-1\right) y-x+\epsilon c_{2}(t)
\end{aligned}
$$

When $c_{1}=0$ and $c_{2}=0$ the equations reduce to a single Van der-Pol oscillator, with a stable limit cycle and an unstable spiral repellor at the origin $\left(x^{*}=0, y^{*}=0\right)$. When $c_{1} \neq 0, c_{2} \neq 0$, the effective dynamics of each oscillator in the network (Eqn. 5) can be seen as an oscillator with fluctuating parameters $c_{1}$ and $c_{2}{ }^{30}$. Note that when $c_{1} \neq 0$ one obtains the fixed point solutions:

$$
\begin{aligned}
& x^{*}=\frac{1 \pm \sqrt{1-4 \mu \epsilon^{2} c_{1}\left(c_{2}-\mu c_{1}\right)}}{2 \epsilon \mu c_{1}} \\
& y^{*}=-\epsilon c_{1}
\end{aligned}
$$

This implies that we get two fixed point solutions for each oscillator in the coupled system. For very weak coupling $(\epsilon \rightarrow 0)$ the additional fixed point sits at infinity. As coupling strength increases this fixed point moves towards the limit cycle, and at some critical coupling strength collides with it, undergoing a saddle node bifurcation. This global bifurcation leads to the destruction of the attracting limit cycle and consequent blow-up. Now for noisy parameters $c_{1}$ and $c_{2}$, such a collision leading to annihilation of the limit cycle occurs for sufficiently large fluctuations in $c_{1}$ and $c_{2}{ }^{30}$. Since the magnitude of fluctuations is governed directly by coupling strength, this implies that blow-ups may occur for sufficiently large $\epsilon$.

In this scenario, the underlying mechanism that controls the blow-up is the following: when the links are changed rapidly (i.e. $r$ is small) to first approximation the terms $c_{1}(t)$ and $c_{2}(t)$ in Eqn. 5 averages to zero, as it is a sum of uncorrelated random inputs. This effectively reduces Eqn. 5 to that of a single Van der Pol oscillator for which there is no blow-up. Alternately, one can argue that for rapid random switching of coupling connections the deviations from the single oscillator dynamics is essentially a sum of uncorrelated contributions, which to first approximation average out to zero.

\section{Synchronization of the Bounded State}

Returning to the space-time plots displayed in Fig. 1, we notice an additional feature. In the figure, two cases with different network rewiring time periods are shown: a fast rewiring case and a slow network change scenario. While there is no blow-up in either case, the emergent bounded state is different for networks rewiring at different rates. When the underlying network changes rapidly a synchronized state is obtained ${ }^{3-35}$, as evident in the representative example in the upper panel of Fig. 1. However, slow network changes yield bounded dynamics that is not synchronized as seen in the representative example in the lower panel of Fig. 1.

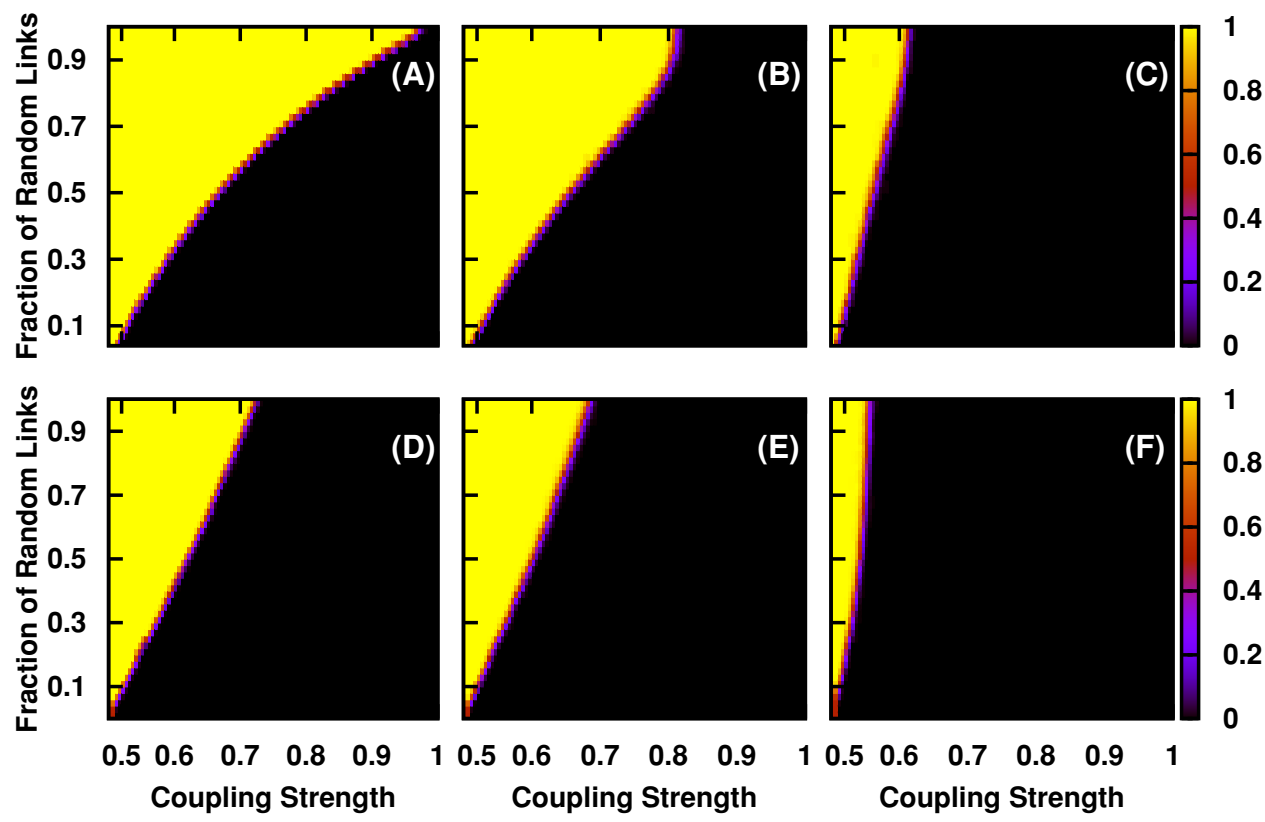

Figure 2| Transition between bounded and unbounded dynamics in a system of 100 coupled oscillators, as reflected in the variation of the boundedness order parameter $Z_{\text {bound }}$ (see text) with increasing coupling strength $\epsilon$ and fraction of random links $p$, for directed networks with network switching time period $r$ equal to (A) 0.01 , (B) 0.1 and (C) 1 , and for undirected networks with $r$ equal to (D) 0.01 , (E) 0.1 and (F) 1 . 
We investigate in detail the synchronization properties of the system. Specifically we attempt to answer the following question: when random coupling prevents a blow-up, does it yield a state that is synchronized, or not? In order to quantify the degree of synchronization in the network we compute an average error function as the synchronization order parameter, defined as the mean square deviation of the instantaneous states of the nodes:

$$
Z=\frac{1}{N} \sum_{i=1}^{N}\left\{\left[x_{i}(t)-\langle x(t)\rangle\right]^{2}\right\},
$$

where $\langle x(t)\rangle$ is the space average at time $t$. This quantity $Z$, averaged over time $N$ and over different initial conditions, is denoted as $Z_{\text {sync }}$. When $Z_{\text {sync }}=0$ we have complete synchronization.

Note that the time needed to reach synchronization is not simply monotonic with respect to fraction of random links ${ }^{36}$. Instead we observe a region, at intermediate values of $p$, where the transience becomes large. This kind of behavior is reminiscent of the long persistence of transience arising for intermediate values of network connectivity $^{37}$. So in our calculation of the relevant order parameters we have left sufficiently long transience $(\sim 15000)$.

It is evident from Fig. 3 that in case of fast changing networks (i.e. $r$ is sufficiently small) the bounded state that arises after random rewiring is also synchronized. This is evident quantitatively through the vanishingly small synchronization error $\left(Z_{\text {sync }} \sim 0\right)$ in networks where blow-ups have been completely suppressed (i.e. $Z_{\text {bound }}=1$ ). On the other hand, for slowly changing networks the synchronization error is significant, even when the dynamics is bounded i.e. $Z_{\text {sync }}$ is not close to zero even when $Z_{\text {bound }}=1$, as evident in Fig. 3B. So infrequent network changes have led to a state which is bounded but not synchronized, i.e. slowly switching random links are effective in reigning in the unbounded dynamics, but they do not manage to produce synchronization.

This relaxation oscillator network then yields three kinds of dynamical states: (i) bounded synchronized motion; (ii) bounded unsynchronized dynamics; and (iii) unbounded dynamics. The nature of the dynamics depends on the interplay of the coupling strength $\epsilon$, rewiring probability $p$ and network switching time $r$.

\section{Probabilistic Switching of Links}

Periodic switching of links occurs in situations where the connections are determined by some global external periodic influence. However this is not the most realistic scenario, as the interaction patterns may not change periodically in time. In such cases we must consider a probabilistic model of link switching, such as in $^{24,38,39}$. In this subsection, we study such randomly switched networks in order to determine if the ability to tame explosive growth is robust to the manner in which the links change. Namely, we verify the generality of above observations by investigating the dynamics on a network whose links switch randomly and asynchronously in time.

Specifically, a link has a probability $p_{r}$ of being rewired (the "link rewiring probability") in some time interval (see methods). So when $p_{r}$ is higher, the links are switched around more often. Note that as before, when a node rewires, it links to its nearest neighbors with probability $1-p$ and to some random site with probability $p$.

The results are displayed in Figs. 4-6. As in the case of periodically changing networks, here too random links manage to control the explosive growth in the system for sufficiently large fraction of random links. When the probability of rewiring the links is larger, namely when the network changes more often, the critical coupling strength at which blow-up occurs is pushed back, yielding a larger region of bounded dynamics.

The interplay of the fraction of random links $p$ and the link rewiring probability $p_{r}$ reveals some interesting scaling relations. Notice first that the critical coupling strength $\epsilon_{c}$ beyond which blow-ups occur, scales with the link rewiring probability $p_{r}$ and fraction of random links $p$ as:

$$
\epsilon_{c} \sim\left(p p_{r}\right)^{\beta}
$$

Fitting the data using the nonlinear least-squares MarquardtLevenberg fitting algorithm yields $\beta=0.119 \pm 0.001$. This is clearly evident quantitatively in the data collapse displayed in Fig. 5A. This scaling relation implies that as the connections change more frequently, and there is larger fraction of random links in the connectivity matrix, the range over which bounded dynamics is obtained becomes larger. Also notice that the effective quantity that is relevant in the scaling relation is the product of $p$ and $p_{r}$, i.e. $p$ and $p_{r}$ may vary, but the emergent phenomena is the same if their product remains the same.

Further calculating the minimum fraction of random links $p_{c}$ necessary to prevent blow-ups, for a fixed coupling strength, at different link rewiring probabilities $p_{r}$ reveals that

$$
p_{c} \sim\left(p_{r}\right)^{\delta}
$$

The results are displayed in Fig. 5B for two different values of coupling. Using the nonlinear least-squares Marquardt-Levenberg fitting

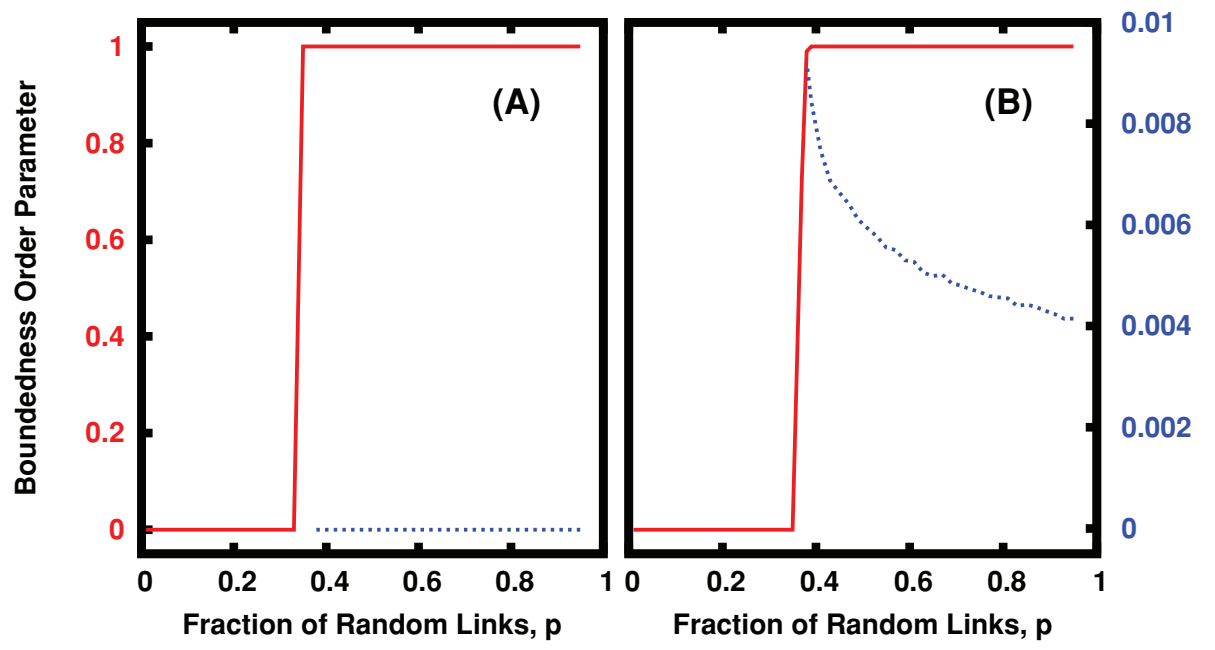

Figure 3 Variation of the Boundedness Order Parameter $Z_{\text {bound }}$ (solid red curve) and the Synchronization Order Parameter $Z_{\text {sync }}$ (dotted blue curve), averaged over 100 initial conditions for varying fraction of random links $p$. Here system size $N=100$, coupling strength $\epsilon=0.6$ and network rewiring time period (A) $r=0.01$ and (B) $r=0.1$. 


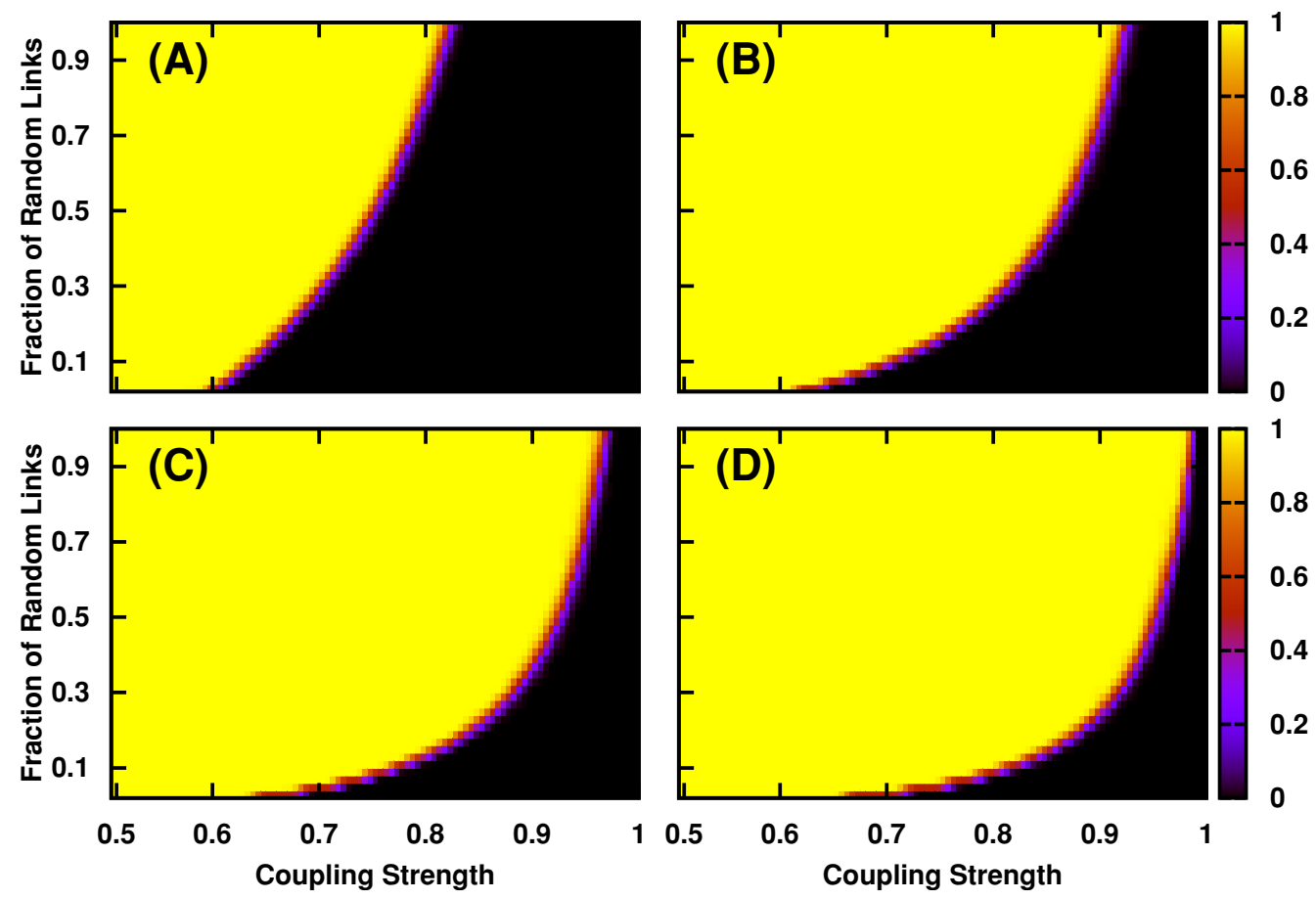

Figure $4 \mid$ Transition between bounded and unbounded dynamics in a system of 100 coupled oscillators with probabilistically switched links, as reflected in the variation of the boundedness order parameter $Z_{\text {bound }}$ (see text) with increasing coupling strength $\epsilon$ and fraction of random links $p$. The link switching probability $p_{r}$ is: (A) $0.1,(B) 0.3$ (C) 0.6 and (D) 0.9 .

algorithm one obtains $\delta=-1.04 \pm 0.008$ for $\epsilon=0.7$ and $\delta=-0.98$ \pm 0.008 for $\epsilon=0.9$.

So when the system has large spatial randomness infrequent changes of links manage to suppress blow-ups, while in a system with few spatially random connections the links need to change more often in order to contain the blow-ups. This again implies (as did the scaling form in Fig. 5A) that blow-ups can actually be tamed at quite small link rewiring probabilities and in networks with small fraction of random links, underscoring the broader relevance of our observations.

Lastly, we observe that for networks that change connections fast (i.e. when $p_{r}$ is large) the bounded state that arises after random rewiring is also synchronized, as evident quantitatively through the vanishingly small synchronization error in these networks (cf. Fig. 6A). However for slowly changing networks (i.e. when $p_{r}$ is small) the synchronization error is quite significant even when the dynamics is bounded (cf. Fig. 6B), namely, slowly changing links yield a state that is bounded but not synchronized.

\section{Generality of our central result}

Finally, in order to further gauge the generality of the above findings we investigated different coupling forms and local dynamics. For instance, we studied some other forms of nonlinear coupling, such as:
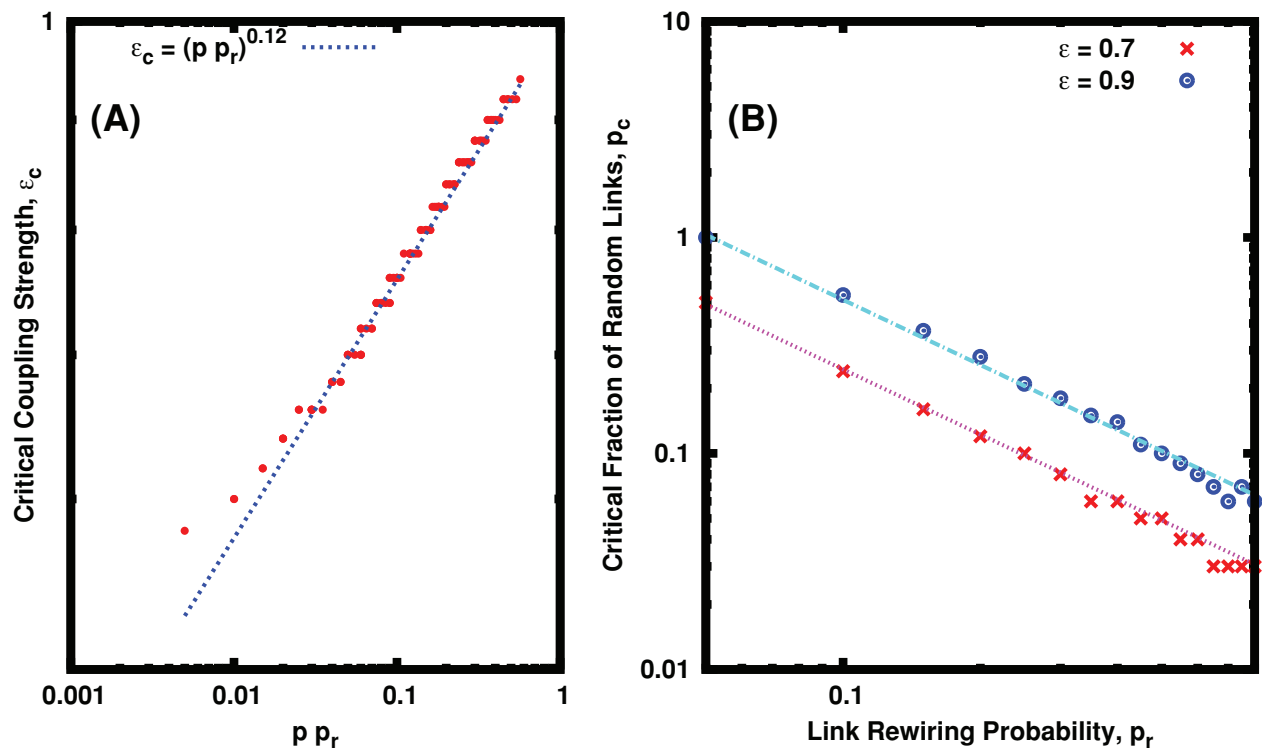

Figure $5 \mid$ (A) Variation of the critical coupling strength at which blow-up occurs, $\epsilon_{c}$, with respect to the product of the link rewiring probability $p_{r}$ and fraction of random links $p$. The points are data from a wide range of $p$ and $p_{r}$, and they collapse to the scaling form shown by the line: $\epsilon_{c} \sim\left(p p_{r}\right)^{\beta}$ with $\beta=$ 0.119. (B) Variation of the minimum fraction of random links necessary to prevent blow-ups vs link rewiring probability $p_{r}$, at coupling strengths $\epsilon=0.7$ (red) and 0.9 (blue). The straight lines show the fit, using the nonlinear least-squares Marquardt-Levenberg fitting algorithm, to the scaling form: $p_{c} \sim p_{r}^{-1}$. 


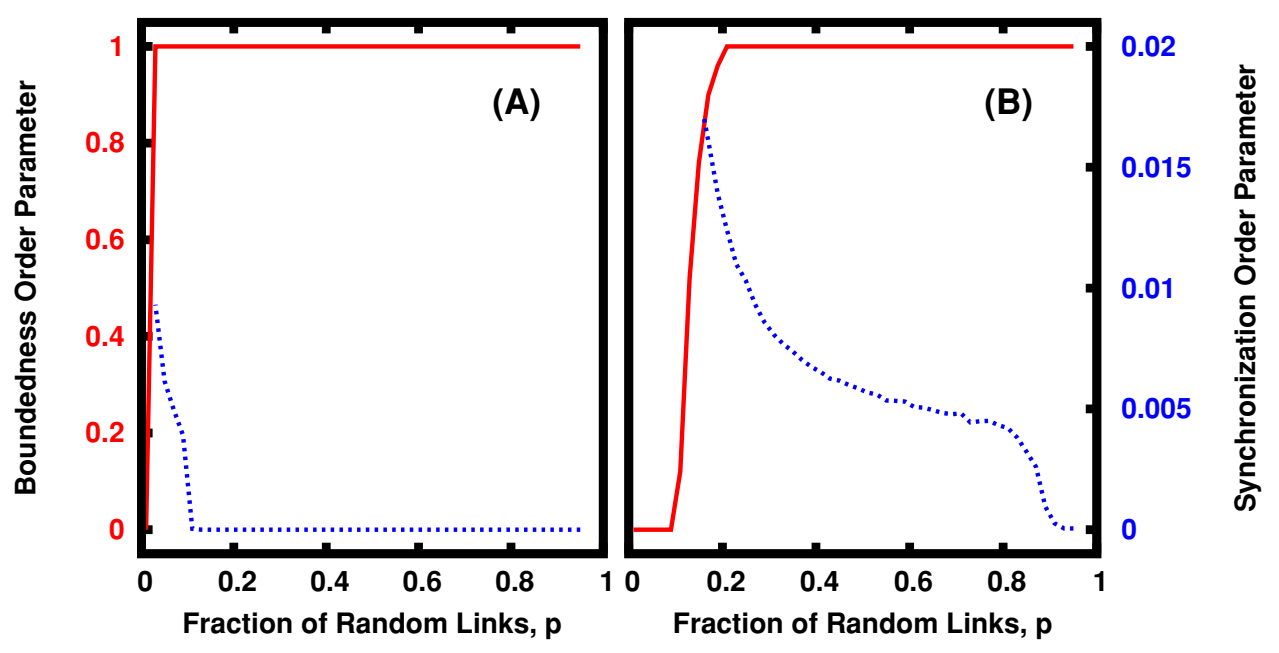

Figure 6 Variation of the Boundedness Order Parameter $Z_{\text {bound }}$ (solid red curve) and the Synchronization Order Parameter $Z_{s y n c}($ dotted blue curve), averaged over 100 initial conditions, with respect to fraction of random links $p$ for link rewiring probability $(\mathrm{A}) p_{r}=0.9$ and $(\mathrm{B}) p_{r}=0.1$. Here system size $N=100$ and coupling strength $\epsilon=0.6$, which is significantly greater than $\epsilon_{c}$.

$$
\begin{aligned}
& \dot{x}_{i}=f\left(x_{i}+\frac{\epsilon}{2}\left[x_{i-1}+x_{i+1}-2 x_{i}\right], y_{i}+\frac{\epsilon}{2}\left[y_{i-1}+y_{i+1}-2 y_{i}\right]\right) \\
& \dot{y}_{i}=g\left(x_{i}+\frac{\epsilon}{2}\left[x_{i-1}+x_{i+1}-2 x_{i}\right], y_{i}+\frac{\epsilon}{2}\left[y_{i-1}+y_{i+1}-2 y_{i}\right]\right)
\end{aligned}
$$

and

$$
\begin{aligned}
& \left.\dot{x}_{i}=f\left(x_{i}, y_{i}\right)+\frac{\epsilon}{2}\left[x_{i-1}^{2}+x_{i+1}^{2}-2 x_{i}^{2}\right]\right) \\
& \left.\dot{y}_{i}=g\left(x_{i}, y_{i}\right)+\frac{\epsilon}{2}\left[y_{i-1}^{2}+y_{i+1}^{2}-2 y_{i}^{2}\right]\right)
\end{aligned}
$$

Now, simulating all these different coupling forms above (Eqns. 10 and 11), with some fraction of links randomized, displayed the same qualitative phenomena: After a certain critical coupling strength we encountered a blow-up in a regular ring of these oscillators, with the blow-ups occurring very early in some systems (e.g. $\epsilon_{c}<0.1$ in Eqn. 11). However as observed in the earlier systems, dynamically randomizing the links in the network suppressed this explosive growth yielding bounded dynamics over a larger range of coupling strengths. Note that the range of the bounded region depends on the specifics of the coupling form and the local dynamics, as well as the fraction and frequency of change of the random links.

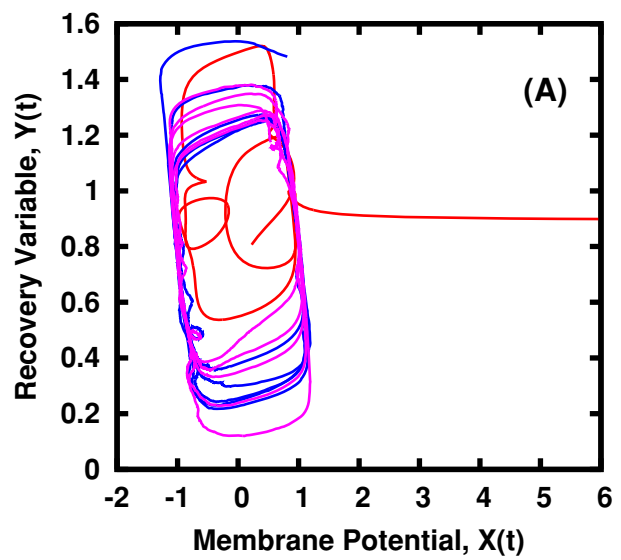

Lastly, we have also investigated the global behavior of the system under different nodal dynamics. For instance, we investigated collections of nonlinearly coupled Stuart-Landau oscillators, where the local dynamics was given by:

$$
\dot{z}=\left(1+i \omega-|z|^{2}\right) z
$$

where $\omega$ is the frequency and $z(t)=x(t)+i y(t)$.

We also studied nonlinearly coupled networks of FitzHughNagumo oscillators, modeling neuronal populations, described by the local dynamics ${ }^{12,13}$ :

$$
\begin{aligned}
& \dot{x}=x-x^{3} / 3-y+I \\
& \dot{y}=\Phi(x+a-b y)
\end{aligned}
$$

where $x$ is the membrane potential, $y$ is the recovery variable and $I$ is the magnitude of stimulus current.

Further, we have explored the heterogeneous case where the nonlinearity parameter $\mu$ is distributed over a range of positive values for the local Van der Pol oscillators.

Our extensive numerical simulations showed qualitatively similar behavior for all of the above systems (see Fig. 7 for representative

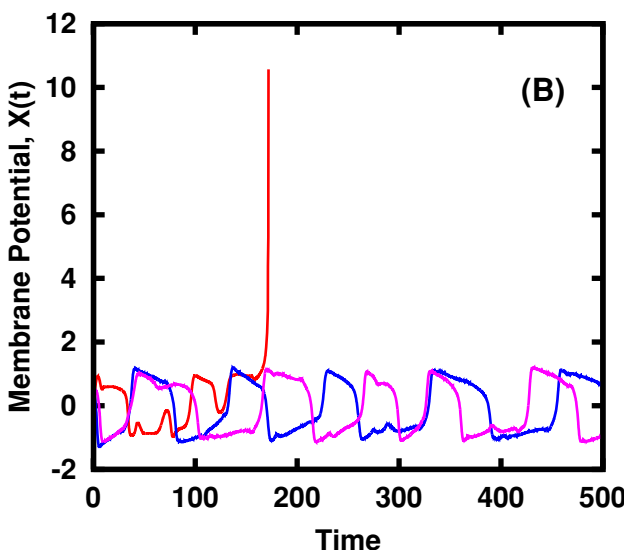

Figure $7 \mid$ (A) Phase portraits and (B) time evolution of the membrane potential, for two representative FitzHugh-Nagumo model neurons in the network given by Eqn. 13, with fraction of random links $p=0.5$, time period of changing the links $r=0.01$, and coupling strength $\epsilon=0.54$ (magenta and blue curves). The case of a static regular network, exhibiting blow-up, is shown alongside as well (red curve). Note that the bounded orbits obtained under dynamic random links are not synchronized (magenta and blue curves). 
examples). This strongly suggests that the phenomenon of prevention of blow-ups through dynamic random links is quite general.

\section{Conclusions}

In summary, we have studied the dynamics of a collection of relaxation oscillators under varying coupling topologies, ranging from a regular ring to a random network. We find that the system blows up under regular ring topology, for sufficiently strong coupling strength. However when some fraction of the links are randomized dynamically, this blow-up is suppressed and the system remains bounded. So our results suggest an underlying mechanism by which complex systems can avoid a catastrophic blow-up. Further from the stand point of potential applications, our observations indicate a method to control and prevent blow-ups in coupled oscillators that are commonplace in a variety of engineered systems.

\section{Methods}

We present the method for changing the connectivity of the network.

\section{Algorithm for periodically switching networks}

1. We start with a 1 dimensional ring with $N$ nodes. Each node $i(i=1, \ldots N)$ has 2 links corresponding to its two nearest neighbors. These regular links of the node are cut with probability $p$ and connected to some randomly selected distant node. In the network, on average, a fraction $p$ of the links are to random nodes and fraction $(1-p)$ are to nearest neighbors. So this network corresponds to a standard small world network ${ }^{7}$ with degree 2 and fraction of random links given by $p$.

2. The above process of generating a small world network of degree 2 and fraction $p$ of random links is repeated after time interval $r$, i.e. the network changes to this new configuration after time $r$. When the connections switch, some nearest neighbor links of the previous network configuration may change to random links and some random links may switch back to regular ones.

So the network switches from one configuration to another periodically, keeping the qualitative nature of the network invariant. Namely, at any instant of time the connectivity matrix has a fraction $(1-p)$ of regular links and fraction $p$ of random links.

\section{Algorithm for stochastically switching networks}

1. We again start with a 1 dimensional ring with $N$ nodes, where each node $i(i=1$, $\ldots N)$ has 2 links. Again fraction $p$ of the links are to random nodes and fraction $(1-p)$ are to nearest neighbors.

2. Now each individual node $i$ has probability $p_{r}$ of changing its links in a specified time interval $\tau$.

3. If the links of a certain node are selected for rewiring, they rewire such that with probability $p$ they connect to random neighbors and with probability $(1-p)$ they connect to nearest neighbors.

In this algorithm, the nodes change their links independently and stochastically. The probability of the new links being random or regular is determined by $p$ as in the first algorithm (and as in the standard small-world scenario). Note that in the first algorithm $r$ is the time period of link changes which occur globally throughout the network, while in the second algorithm uncorrelated changes occur at the local level.

Our simulations show that these two very different ways of varying the network both yield the same qualitative phenomena: namely changing links helps to suppress blow-ups in the system.

Method for simulating the dynamics of the system. We solved the $2 \mathrm{~N}$ coupled nonlinear ordinary differential equations (ODE), given by Eqns. 4, 10, 11 by integrating the ODEs with the fourth order Runge-Kutta algorithm. We have checked that the results are qualitatively same over a large range of step sizes $\left(\sim 10^{-3}\right.$ to $\left.10^{-7}\right)$, in order to rule out artefacts arising from the stiffness problem in such differential equations.

We obtained the shapes and sizes of the limit cycles arising in this network, for system sizes ranging from $N=10$ to $N=10^{3}$, under varying fraction of random links $p(0 \leq p \leq 1)$. We investigated a large range of network switching time periods $r(0.01$ $\leq r \leq 1)$ for the case of periodically switched networks, and $0 \leq p_{r} \leq 1$ for the case of stochastically switched networks (with $\tau=0.001$ ).

System parameters. In the system given by Eqns. 3 and 4 we have investigated a range of nonlinearity parameters $\mu$ and we present the results for the representative case of $\mu$ $=1.5$ in the examples displayed in this paper. In the system given by Eqn. 13, parameters $\Phi, a, b$ are taken to be $0.08,0.7,0.8$ respectively.
3. Perez, G., Pando-Lambruschini, C., Sinha, S. \& Cerdeira, H. A. Nonstatistical behavior of coupled optical systems. Phys. Rev. A 45, 5469-5473 (1992).

4. Zanette, D. H. \& Mikhailov, A. S. Condensation in globally coupled populations of chaotic dynamical systems. Phys. Rev. E 57, 276-281 (1998).

5. Wang, W., Kiss, I. Z. \& Hudson, J. L. Experiments on arrays of globally coupled chaotic electrochemical oscillators: Synchronization and clustering. Chaos 10, 248 (2000).

6. Barrow, J. D. The Infinite Book: A Short Guide To The Boundless, Timeless And Endless (Vintage, 2005).

7. Watts, D. J. \& Strogatz, S. H. Collective dynamics of small-world networks. Nature 393, 440-442 (1998)

8. Pecora, L. M. \& Carroll, T. L. Master stability functions for synchronized coupled systems. Phys. Rev. Lett. 80, 2109-2112 (1998)

9. Van der Pol, B. On relaxation-oscillations. Phil. Mag. 2, 978-992 (1926).

10. Van der Pol, B. \& Van der Mark, J. Frequency demultiplication. Nature 120, 363-364 (1927).

11. Van der Pol, B. \& Van der Mark, J. The heartbeat considered as a relaxation oscillation, and an electrical model of the heart. Phil. Mag. 6, 763-775 (1928).

12. FitzHugh, R. Impulses and physiological states in theoretical models of nerve membrane. Biophys. J. 1, 445-466 (1961).

13. Nagumo, J., Arimoto, S. \& Yoshizawa, S. An active pulse transmission line simulating nerve axon. Proc. Ire. 50, 2061-2070 (1962).

14. Cartwright, J. H., Eguiluz, V. M., Hernandez-Garcia, E. \& Piro, O. Dynamics of elastic excitable media. Int. J. Bifur. Chaos 9, 2197-2202 (1999).

15. Prasad, A., Dhamala, M., Adhikari, B. M. \& Ramaswamy, R. Amplitude death in nonlinear oscillators with nonlinear coupling. Phys. Rev. E 81, 027201 (2010).

16. Fitzgibbon, W. E. \& Walker, H. F. Nonlinear diffusion (Pitman, 1977).

17. Friston, K. J. Book Review: Brain Function, Nonlinear Coupling, and Neuronal Transients. The Neuroscientist 7, 406-418 (2001).

18. Chen, C.-C. et al. Nonlinear Coupling in the Human Motor System. J. of Neurosci. 30, 8393-8399 (2010)

19. Muller-Stoffels, M. \& Wackerbauer, R. Regular network model for the sea icealbedo feedback in the Arctic. Chaos 21, 013111 (2011).

20. Pastor, I., Perez-Garcia, V. M., Encinas, F. \& Guerra, J. M. Ordered and chaotic behavior of two coupled van der Pol oscillators. Phys. Rev. E 48, 171-182 (1993)

21. Belykh, I. G., Belykh, V. N. \& Hasler, M. Blinking model and synchronization in small-world networks with a time-varying coupling. Physica D 195, 188206 (2004).

22. Sinha, S. Random coupling of chaotic maps leads to spatiotemporal synchronization. Phys. Rev. E 66, 016209 (2002).

23. Mondal, A., Sinha, S. \& Kurths, J. Rapidly switched random links enhance spatiotemporal regularity. Phys. Rev. E 78, 066209 (2008).

24. Kohar, V. \& Sinha, S. Emergence of epidemics in rapidly varying networks. Chaos, Solitons \& Fractals 54, 127-134 (2013).

25. Varela, F., Lachaux, J. P., Rodriguez, E. \& Martinerie, J. The brainweb: phase synchronization and large-scale integration. Nat. Rev. Neurosci. 2, 229-239 (2001).

26. Brezina, V., Orekhova, I. V. \& Weiss, K. R. The neuromuscular transform: the dynamic, nonlinear link between motor neuron firing patterns and muscle contraction in rhythmic behaviors. J Neurophysiol. 83, 207-231 (2000).

27. Von der Malsburg, C. Handbook of Brain Theory and Neural Networks, [Dynamic Link Architecture, p.365] (MIT Press, 2002).

28. Sturis, J. \& Brøns, M. Local and global bifurcations at infinity in models of glycolytic oscillations. J. Math. Biol. 36, 119-132 (1997).

29. Abraham, R. H. \& Bruce Stewart, H. A chaotic blue sky catastrophe in forced relaxation oscillations. Physica D 21, 394-400 (1986).

30. Hong, L. \& Sun, J. Q. A fuzzy blue sky catastrophe. Nonlinear Dynamics 55, 261-267 (2009)

31. McCann, K. \& Yodzis, P. Nonlinear dynamics and population disappearances. Amer. Nat. 144, 873-879 (1994).

32. Ghosh, D., Banerjee, S. \& Chowdhury, A. R. Synchronization between variable time-delayed systems and cryptography. Euro. Phys. Letts. 80, 30006 (2007).

33. Barahona, M. \& Pecora, L. M. Synchronization in small-world systems. Phys. Rev Lett. 89, 054101 (2002)

34. Matthews, P. C., Mirollo, R. E. \& Strogatz, S. H. Dynamics of a large system of coupled nonlinear oscillators. Physica D 52, 293-331 (1991)

35. Hong, H., Choi, M. Y. \& Kim, B. J. Synchronization on small-world networks. Phys. Rev. E 65, 026139 (2002).

36. Poria, S., Shrimali, M. D. \& Sinha, S. Enhancement of spatiotemporal regularity in an optimal window of random coupling. Phys. Rev. E 78, 035201 (2008).

37. Zumdieck, A., Timme, M., Geisel, T. \& Wolf, F. Long chaotic transients in complex networks. Phys. Rev. Lett. 93, 244103 (2004).

38. Vazquez, F. \& Zanette, D. H. Epidemics and chaotic synchronization in recombining monogamous populations. Physica D 239, 1922-1928 (2010).

39. Zanette, D. H. \& Gusmán, S. R. Infection spreading in a population with evolving contacts. J. Biol. Phys. 34, 135-148 (2008).

\section{Acknowledgments}

V. Kohar acknowledges senior research fellowship (SRF) from Council of Scientific \& Industrial Research (CSIR), India.
Scientific, Singapore, 1987)

2. Wiesenfeld, K., Colet, P. \& Strogatz, S. H. Synchronization transitions in a disordered Josephson series array. Phys. Rev. Lett. 76, 404-407 (1996). 


\section{Author contributions}

S.S. conceived the problem, and A.C. and V.K. performed all the numerical simulations. S.S., A.C. and V.K. discussed the results and wrote the manuscript together.

\section{Additional information}

Competing financial interests: The authors declare no competing financial interests.
How to cite this article: Choudhary, A., Kohar, V. \& Sinha, S. Taming Explosive Growth through Dynamic Random Links. Sci. Rep. 4, 4308; DOI:10.1038/srep04308 (2014).

(c) (1) (2) This work is licensed under a Creative Commons Attribution-
NonCommercial-ShareAlike 3.0 Unported license. To view a copy visit http://creativecommons.org/licenses/by-nc-sa/3.0 